\title{
INDEX OF DIARIES EXTENDING OVER MORE THAN TEN YEARS
}

\begin{tabular}{l} 
1504-1588, Lambarde \\
1532-1605, Birrel \\
1539-1613, Parry \\
1544-1608, Young \\
1550-1563, Machin \\
1564-1602, Forman \\
1570-1617, Mildmay \\
1570-1583, Walsing- \\
\multicolumn{1}{c}{ ham } \\
1571-1610, Melville \\
1577-1601, Dee \\
1586-1608, Wyot \\
1592-1603, Henslowe \\
1592-1643, Laud \\
1593-1616, Wilbraham \\
1595-1648, Oglander \\
1595-1629, Ward \\
1595-1610, Winthrop \\
1597-1614, Casaubon \\
1603-1654, Powell \\
1604-1641, Montague \\
1604-1628, Yonge \\
1607-1651, Derby \\
1608-1633, Ayshcombe \\
1611-1643, Cork \\
1616-1645, Young \\
1618-1634, Whiteway \\
1621-1641, Herbert \\
1622-1646, Roberts \\
1624-1647, Forbes \\
1625-1642, Rous \\
1626-1654, Crosfield \\
1628-1645, Burghall \\
\end{tabular}

$1630-165^{8}$, Wallington

1632-1643, Dugard

1632-1660, Wariston

1633-1688, Ashmole

1633-1646, Hope

I633-1651, Mildmay

$1634-1672$, Wemyss

1635-1659, Greene

1636-1680, Whittingham

1637-1662, Baillie

1638-1648, Jeffries

1638-1648, Slingsby

1640-1654, Cuningham

1640-1706, Evelyn

I640-1688, Sanderson

1643-1686, Dugdale

1644-1681, Josselin

1644-1655, Penn

1646-I66I, Leicester

1648-1679, Ward

1649-166I, Harcourt

1649-1671, Lamont

I649-1688, Mainwaring

1650-168, Henry

1650-1683, Jackson

1650-1667, Nicoll

1652-1680, Brodie

1652-1663, Johnson

1654-1689, Reresby

1655-1668, Gordon

1655-1679, Moore

1656-1678, Mordaunt
1657-1695, Wood

1659-1703, Barlow

1659-1672, Rugge

I660-1678, Allin

1660-I68o, Blundell

1660-1676, Brownlow

1661-1722, Borland

1661-1724, Gardiner

1663-1674, Lowe

1664-1717, Newton

I665-1676, Lauder

I665-I725, Pledger

1665-1692, Roch

1665-1677, Stockton

1666-1702, Heywood

1666-1705, Leeds

1666-1703, Yonge

1667-1676, Anglesey

1668-1708, Smith

I668-1697, Whitley

1671-1709, Brockbank

1671-1714, Freke

I671-1693, Jolly

1671-1721, Sitwell

1672-170r, Sampson

1674-1723, Lee

1675-1693, Brown

1675-1704, Locke

1677-1697, Bohun

1677-1699, Bufton

1677-1724, Thoresby

I678-I724, Luttrell

1679-1691, Dampier

[ xxi] 


\begin{tabular}{|c|c|c|}
\hline \multicolumn{3}{|c|}{ Diaries of More than Ten Years } \\
\hline 1679-1709, Lowndes & I700-1716, Edgar & I723-1779, Bagshaw \\
\hline 1680-1692, Anon. & I70I-1755, Clegg & $1723-1764$, Howard \\
\hline 1680-1706, Newdigate & I70I-I743, Kelsall & $1723-173^{8}$, Oxford \\
\hline 1681-I702, Aston & I701-1712, Millner & 1725-1795, Butterfield \\
\hline $1681-1707$, Bee & I70I-1731, Wodrow & $1725^{-1744}$, Gordon \\
\hline 1682-1724, Stapley & $1702-1713$, Sharp & $1725-1735$, Hobson \\
\hline $1683^{-1717}$, Burrell & $1703-1716$, Briggins & $1725^{-1756, \text { Purefoy }}$ \\
\hline 1684-1725, Nicolson & I703-1713, St. Pierre & $1725^{-1791, ~ W e s l e y ~}$ \\
\hline 1685-I699, Richards & I704-I730, Isham & (John) \\
\hline 1686-1704, Turnbull & I705-I735, Hearne & $1727-176_{3}$, MacDonald \\
\hline I687-I7ro, Petiver & I706-I731, Sanderson & I729-1751, Doddridge \\
\hline 1688-1742, Bristol & 1707-1737, Bell & $1729-1764$, Stukeley \\
\hline 1688-r704, Nasmyth & 1707-1719, Booth & 1731-1794, Mascall \\
\hline 1688-1708, Granger & 1707-1722, Erskine & I73I-I747, Vertue \\
\hline I688-I699, Rokeby & 1707-1740, Nasmyth & 1733-1760, Monteage \\
\hline 1689-1707, Kaye & I707-1743, Norris & r734-1760, Bulkeley \\
\hline I689-I743, Stapley & I708-I730, Anon. & 1734-1749, Ingham \\
\hline I690-1702, (Cromwell) & I708-1738, Rees & 1736-1756, Wesley \\
\hline 1690-1720, Murray & $1709-1726$, Morris & (Chas.) \\
\hline I690-I705, Richards & $1709-1720$, Rud & I736-1770, Whitefield \\
\hline 1692-I722, Calverley & 1711-1732, Housman & $1737^{-1750, ~ K a y ~}$ \\
\hline $1692-1729$, Coe & I7I I-I729, Walkden & $1737^{-1752, ~ W e s t o n ~}$ \\
\hline 1693-1720, Bury & 171 I-1762, Wright & $173^{8-1803, \text { Mill }}$ \\
\hline 1694-17r6, Anon. & I712-1757, Pace & $1739-1769$, Gray \\
\hline 1694-1732, Savage & I713-1773, Capps & 1740-1753, Anon. (2) \\
\hline I695-1729, Le Neve & I714-1728, Marchant & 1741-1769, Hubbard \\
\hline I6g6-1754, Clerk & $1715-175^{\circ}$, & 1741-1 761, Melcombe \\
\hline I696-i714, Henry & $1715^{-1726, V}$ & 1741-1821, Oliver \\
\hline I696-1713, Newcome & 1717-I755, I & 1742-1752, Bennet \\
\hline I697-1719, Bonhereau & I718-I760, Fretwell & 1744-1757, Beswick \\
\hline I697-1707, Hume & I720-I780, Crosier & I744-I812, Florry \\
\hline 1698-1729, Compton & 1720-1747, Rogers & I 744-I 784 , Ryland \\
\hline 16g8-1724, Gordon & 1720-1731, Wither & 1745-1769, Darby \\
\hline 1699-I709, Evans & 1721-1756, Bayly & I747-1773, Gough \\
\hline 1700-I 728, Blackader & I721-1749, Walker & $174^{8}-1763$, Boscawen \\
\hline $1700-1745$, Brown & I722-1744, Byrom & I748-1796, Douglas \\
\hline
\end{tabular}


1748-1778, Gyll

1749-1769, Gisborne

1750-1779, Baker

1750-1784, Johnson

175 I-1763, Garrick

1751-1763, James

1751-1783, Orford

1751-1797, Palmer

1751-1793, White

1752-1774, Northum-

berland

1752-1775, Stevenson

1753-1777, Glynne

1753-1774, Rutty

1754-1765, Turner

1755-1799, Downing

1755-1814, Hervey

I755-I79I, Neale

1755-1807, Wharton

1756-1800, Bray

1756-1791, Coke

1756-1816, Hamilton

1756-1786, Hoskyns

1756-1808, Powys

1756-1770, Raper

I757-1821, Thrale

1758-1806, Knight

1758-1802, Woodforde

I759-1780, Downman

1759-1770, Hollis

x760-1773, Fawcett

1761-1772, Anon.

1761-1773, Cooke

1761-1802, Fox

$1762-1775$, Thomas

1762-1776, Wigham
1764-1794, Boswell

1764-1826, Gray

1764-1799, Mewburn

1765-1794, Wale

1766-1777, Glynne

1767-1806, Anon.

1767-1792, Ball

1767-1796, Brown

1767-1832, Cullum

1767-1807, Malmesbury

1767-1794, Savage

1767-1792, Sharp

1767-1860, Wright

1768-1779, Cook

1768-1833, D'Arblay

1768-1783, Glenorchy

I768-1810, Maxwell

1768-1788, Prior

1768-1791, Stacpoole

1768-1814, Tompkins

1769-1826, Capper

1769-1808, Clark

1769-1783, Parnell

1769-1810, Windham

1770-1782, Cooper

1770-1830, Day

I770-1831, Jenkins

1770-1820, Wheatley

1770-1797, Wilkes

I771-1785, Ellis

1771-1828, James

1772-1814, Fletcher

1772-1790, Grubb

1772-1797, Humphrey

1772-1829, Lewin

1772-1821, Woods
1773-1789, Boone

1773-1791, Burn

1773-1795, Pedley

1773-1786, Roberts

1774-1814, Bradburn

1774-1790, Dillwyn

1774-1784, Goddard

1774-1790, Hough

1774-1788, Hutchinson

1774-1821, Jones

1774-1826, Leadbeater

1774-1787, Mydleton

1775-1792, Blaikie

1775-1794, Chichester

1775-181 I, Cullum

1775-1800, Laugher

1775-1785, Oxnard

1775-1809, Roe

1775-1795, St. Patrick

1776-1795, Romney

1776-1848, Times

1776-1818, Young

$1777^{-1788, \text { Abell }}$

1778-1812, Charles

1778-1802, Longden

1778-1806, Scott

I779-1826, Belsham

1779-1801, Bishop

1779-1793, Carter

1779-1817, Cussons

1779-1846, Frampton

1779-1818, Hastings

1779-1832, Tindall

1780-1801, Clarke

1780-1796, Temple

1780-1798, White 
xxiv

1781-1832, Clarke

1781-1815, Cooper

1781-1845, Dyott

1781-1833, Knight

1781-1798, Moses

1781-1797, Ord

1781-1813, Stanley

1781-1794, Torrington

I782-1816, Ashworth

1782-181o, Faviere

1783-1848, Berry

1783-1826, Conran

1783-1806, Hordern

1783-1833, Wilberforce

1784-1840, Entwisle

1784-1838, Jones

1784-1797, Pilkington

1784-1809, Rathbone

1785-1815, Burgess

1785-1795, Crewe

$1785-1836$, Cumberland

1785-1809, Meek

1785-1797, Trimmer

1786-1801, Anon. (1)

1786-1821, Anon. (2)

1786-180o, Grape

1787-1799, Bower

1787-1797, Cochrane

1787-1835, Jesup

1787-1833, Losh

1787-1809, Ryan

1788-1842, Allen

1788-1829, Colchester

1788-1821, Farington

1788-1832, Skinner

1789-180r, Elliott
1789-1798, Heron

1789-1820, Marten

1789-1845, Nairne

1789-1808, Steuart

1789-1814, Walker

1789-1820, Wynne

(Eliz.)

1789-1811, Wynne

(Eug.)

1790-1803, Bird

1790-1846, Cowley

1790-1829, Plumptre

1790-1813, Steadman

I790-1816, Wakefield

1790-1832, Wilkinson

1791-1824, Bagshawe

I79I-I803, Clarendon

1791-1802, Cobb

I791-I828, Crabb

I79I-18II, Holland

I791-1812, Poole

1791-1805, Waring

1791-1840, Young

1792-1807, Blore

I792-1850, Brerton

1793-1819, Anon. (I)

I793-I818, Bagshaw

I793-1819, Glenbervie

1793-1813, Heywood

1793-1814, Hoare

1793-1808, Moore

I793-1807, Ompteda

1793-1814, Pearson

I793-1838, Potter

1793-1832, Shillitoe

1794-1809, Cooke
1794-1819, Cope

1794-1839, Day

1794-1818, Hastings

1794-18Ig, Hodgkin-

son

1794-1809, Stewart

1795-1832, Anon. (1)

1795-1812, Danvers

1795-1860, Darling

1795-1816, Marsden

I795-186I, Terry

1795-1814, Thornton

1795-1832, White

1796-1811, Beard

1796-1827, Cary

1796-181 I, Green

1796-1832, Kilham

I796-1815, Miller

1796-1850, Nelson

1797-1845, Fry

1797-1860, Hunter

1797-1818, Kirk

1797-1818, Robertson

1797-1844, Wynn

1798-1810, Carrington

1798-1846, Catchpool

1798-1816, Dymond

1798-1854, Forster

1798-1824, Neville

1798-1830, Page

1798-1827, Trench

I798-1828, Words-

worth

1799-1852, Berry

1799-18 r, Dow

I799-1815, Gomm 
Diaries of More than Ten Years

1799-1811, Hoghton

1799-1829, Maton

1799-1810, Taylor

1799-1810, Thompson

1799-1817, Titherton

1800-1854, Burgoyne

1800-1813, Clark

1800-1810, Fenton

1800-1840, Gibbons

1800-1851, Gisborne

1800-1823, Leyden

1800-1832, Roe

1800-181 I, Rose

$1800-1871$, Ward

1800-1816, Wilson

180r-r829, Addison

1801-1836, Cumberland

I80I-1821, Grenfell

180I-1815, Jackson

I80I-1840, Malmesbury

180I-1832, Porter

180I-1821, Wilmot

1802-1853, Hawker

$1802-1846$, Lucas

1802-1814, Maude

I802-1818, Romilly

1802-1845, Smart

1803-1852, Bourne

1803-1828, Chalmers

1803-1821, Francis

1803-1822, Richmond

1803-1842, Toase

1803-1831, Walker

1804-1849, Backhouse

1804-1859, Calvert
1804-1866, Everett 1804-I846, Heugh

1804-1839, Tomlinson

1805-1816, Anon.

1805-1844, Colquhoun

1805-1818, Cooke

I805-1849, Field

1805-1836, Hall

1805-1830, Mackintosh

1805-1827, Palmer

1805-1816, Ross

1805-1821, Simpson

1805-1820, Wesley

1806-1827, Ashburton

1806-1818, Budgett

1806-1842, Fox

1806-1853, Rastrick

1807-I849, Dundonald

1807-r847, Hardy

1807-1821, Lee

$1807-1832$, Leslie

1807-I862, Raffles

1807-1825, Weeton

1808-1846, Gurney

1808-1868, Harris

I808-1844, Palmerston

1808-I834, Pechell

I808-1842, Richardson

1808-1835, Rowntree

I808-1822, Wellesley-

Pole

I808-1840, Wilkie

I809-I833, Bedingfield

1809-1837, Broadbelt

1809-1852, Broughton

I809-18Ig, Durnford
I809-1850, Erskine

I809-1899, Foley

1809-1871, Griffin

1809-1862, Stanley

1809-1820, Thomas

I809-1820, Ward

1810-1820, Bury

I810-1850, Croker

I810-1860, Hall

1810-1851, Napier

I810-1860, Paul

I810-1849, Tuke

1811-1842, Clift

1811-1835, Crosse

I81 I-1831, Curry

I8I I-I868, Mogg

I8I I-I82I, Pickmore

1811-1867, Robinson

1811-1838, Taylor

I81 I-1858, Yeardley

1812-1895, Beaver

1812-1846, Collins

1812-1837, Dudley

1812-1840, Heywood

1812-1883, Montefiore

I812-1828, Natt

1812-1845, Piggott

I812-1828, Scott

1813-1849, Ellis

I813-1841, Faraday

1813-1830, Finch

1813-1838, Holtzapfel

1813-1837, Strutt

1813-1848, Thackwell

1814-1832, Barclay

1814-1837, Barker 
xxvi

1814-1860, Greville 1814-1839, Hagger I814-1838, James 1814-1840, Shelley (Mary)

I814-1828, Todd 1814-1856, Watkin I815-1867, Bailey 1815-1902, Hennell 1815-1867, Sharples 1815-1857, Ticknor I8I6-1885, Beaumont 1816-1877, Hill 1816-1840, Lister 1816-1873, Turner 1817-1833, Carvosso 1817-1858, Lyell 1818-1839, Anon. (4) 1818-1829, De La Beche I818-1843, Evans 1818-1830, Holland 1818-1857, Jackson 1818-1847, Moore 1818-1842, Perry 1818-1834, Timms 1819-1852, Clinton 1819-1843, Fox 1819-1872, Madden 1819-1852, Mantell I819-I869, Pasley 1819-1829, Selwyn 1819-1863, Sorby 1819-1850, von Neumann

I819-1831, Wythe 1820-1855, Bagot

Diaries of More than Ten Years

1820-1853, Baker 1820-1840, Bosanquet $1820-1876$, Coleridge 1820-1875, Ford 1820-1846, Haydon 1820-1850, Johnston 1820-1833, Kitto 1820-1 855 , Pearson 1820-1863, Romilly 1820-1834, Shilton 1820-1847, Wilson 1820-1860, Wood 1821-1837, Barrett 1821-1832, Cobbett I821-I849, Copleston I82I-I847, Eld I822-1833, Howard 1822-1876, Keppel I822-1836, Knill 1822-I853, Lockhart 1822-1879, Sopwith 1822-1865, Waddy I823-1847, Anon. (2) 1823-1868, Epps I823-1860, Fry I823-1859, Kemp 1823-1833, Palmer 1823-1837, Taylor 1824-1839, Bannerman 1824-1868, Buckley 1824-1852, Capper 1824-1843, Fox 1824-1837, Glynne (Mary) I824-I842, Merivale 1824-1837, Robson
I824-I84I, White 1825-1891, Babington I825-1861, Craik 1825-1874, Glynne 1825-1867, Mewburn 1825-1859, Owenson 1825-1840, Ramsay I825-1858, Russell 1825-1885, Shaftesbury I826-1865, Alexander I826-1870, Alford 1826-1895, Gladstone 1826-1840, Webb 1827-1837, Curling I827-1853, Opie 1827-184I, Pamplin I828-1892, Bonar 1828-1855, Hamilton 1828-1877, Parry I828-1882, Parsons I828-1844, Stanley I828-I884, Tregelles 1829-1842, Cameron 1829-1846, Finlay 1829-1876, Gregory 1829-1863, Griffith 1829-1877, Hall I829-I882, Leyland 1829-1879, Murphy 1829-1853, Sedgwick I829-I848, Shaw 1829-1872, Young 1830-1859, Braithwaite 1830-I842, Burney 1830-1892, Mueller 1830-I840, Rathborne 


\section{Diaries of More than Ten Years}

1830-1898, Russell

1830-1858, Russell

1830-1841, Scott

1830-1852, Sharp

$1830-1870$, Shee

1830-1862, Vignoles

1830-1873, Wilberforce

1831-1854, Cockburn

183I-1866, Forster

1831-1842, Gross

1831-1847, Keith

1831-1884, Pattison

1832-1853, Blakey

1832-1903, Cambridge

1832-1872, Greville

I832-1858, Herford

1832-1844, Hodgson

1832-1842, Lacaita

I832-1869, Malmesbury

I832-1847, Raikes

1832-1882, Victoria

$1833-1857$, Fry

1833-1872, MacLeod

1833-1851, Macready

1833-1890, Palgrave

1833-1884, White

1834-1885, Bunbury

I834-1851, Jenkins

1834-1873, Morgan

1834-1873, Owen (bis)

1834-1854, Rees

I $834-1852$, Wicksteed

I835-1849, Allen

I835-1892, Connal

1835-1856, Ewing

I835-1867, Fox
1835-1850, Layard

1835-1855, Mends

I835-I862, Tanner

I835-1891, Winstanley

1836-1866, Ellis

1836-1875, Halliwell-

Phillipps

1836-1871, Rees

1837-1852, Addams-

Williams

1837-1847, Backhouse

1837-1870, Bacon

I837-I887, Bright

I837-1864, Cavendish

1837-I859, Chatterton

1837-1887, Claughton

1837-1878, De Rothschild

1837-1859, Murray

1837-1857, Powell

1837-1948, Soame

1837-1851, Southall

I838-1885, Harris

1838-1848, Hughes

1838-1859, Macaulay

$1838-1857$, Pease

1838-1864, Roberts

1838-1882, Sargent

1838-1899, Stone

1839-1852, Borradaile

1840-1875, Daniel

1841-1904, Cranbrook

1841-1891, Fowler

1842-I89I, Cramp

1842-1888, Daunt

I842-1866, Hopkins
1842-I862, Roffe

I842-1857, Wire

1843-1894, Braithwaite

1843-1867, Brightwell

I843-1864, Carlisle

1843-1859, Goodall

I843-I862, Jeune

I843-I902, Pollen

I844-1888, Blanchard

1844-1856, Brown

1844-1874, Cowell

1844-1862, Dalton

1844-I890, Manning

1844-1866, Nichols

1844-1874, Pollock

1844-I885, Watkins

1845-1869, Dennis

I845-I870, Griffith

I845-ı868, Lear

1846-1870, Collinson

1847-1889, Allingham

I847-I86I, Campbell

1847-1857, Cope

1847-1882, Harford-

Battersby

1847-1873, Pearson

1847-1880, Redgrave

I847-1882, Wedder-

burn

1848-1876, Davies

1848-1869, Hanbury

1848-1894, Hornby

I848-1876, Kenealy

1848-1859, Robson

1848-1858, Senior

1848-1885, Sewell 


\section{xxviii}

\section{Diaries of More than Ten Years}

\begin{tabular}{|c|c|c|}
\hline I848-1887, Shields & $1856-1870$, Williams & 1866-1923, Daniel \\
\hline 1849-1859, Beale & $185^{6}-1912$, Wrench & 1866-1916, Davies \\
\hline 1849-1868, Ponsonby & $1857-188_{3}$, Bain & 1866-I939, Henson \\
\hline 849-1870, Rossetti & 1857-1905, Champness & 1866-1880, Iddesleigh \\
\hline I850-I860, Miers & 1857-1915, Chancellor & I868-r878, Dawson \\
\hline 1850-I906, Raven & 1857-1891, McMullen & 1868-1900, Dickson \\
\hline 1850-1893, Thorold & 1857-1869, Moore & $1869-1895$, Chichester \\
\hline $850-1903$, Vivian & $1857-1871$, Ossington & $1869-1885$, Schreiber \\
\hline $851-1874$, Daly & $1857-1880$, Ramsay & $1870-1890$, Beers \\
\hline 1851-1901, Grant Duff & $185^{8-1912, ~ R u d d y}$ & 1870-1914, Blunt \\
\hline $1851-1875$, Moran & 1859-1872, Amberley & 1870-1906, Liddell \\
\hline 851-I866, Morley & 1859-1869, Frith & 1870-1907, Ribblesdale \\
\hline 851-1892, Pamplin & 1859-1887, Thring & 1871-1894, Fitzpatrick \\
\hline 1851-1932, Salter & 1860-Igro, Batty, & 187I-I893, Head \\
\hline $185^{2-1866, ~ J e v o n s ~}$ & (Mrs. B.) & 187I-I900, Lucas \\
\hline $185-1874$, Stevenson & 1860-1872, Cameron & $1872-1885$, Brown \\
\hline 853-1894, Dodgson & I860-I880, Peacock & 1872-1918, Esher \\
\hline 853-1866, Fox & 1860-1923, Sadler & 1873-1903, Bosanquet \\
\hline $853-1867$, Jones & I860-1888, Symonds & 1873-1893, Davidson \\
\hline 1853-1908, Kemp & $1861-1872$, Batty & 1873-1895, Monkswell \\
\hline 1853-1863, Richard & $186 I-1871$, Vincent & 1875-19I9, Courtney \\
\hline $854-1875$, Amberley & 1862-1914, Butler & 1876-1900, Goodsall \\
\hline $854^{-1880, \text { Cavendish }}$ & 1862-I887, Campbell- & 1876-1945, Thompson \\
\hline I854-1873, Glasgow & Bannerman, & 1877-1921, Cook \\
\hline I854-I9ro, Griffith & (Lady) & $1877-1887$, Jefferies \\
\hline 1854-1874, Ritchie & 1862-1875, Hopkins & 1877-1890, Schlüter \\
\hline $854-1888$, Walker & $1862-1876$, Rees & 1878-1903, Anon. \\
\hline $855-1865$, Burgess & $1862-1900, \mathrm{Sim}$ & 1878-1896, Benson \\
\hline 1855-1908, Campbell- & 1862-1890, Slingsby & 1878-1907, Collins \\
\hline Bannerman & 1863-1873, Cory & 1878-1900, Evans \\
\hline I855-1879, Eliot & 1863-1885, Hannington & 1878-1894, Hughes \\
\hline I855-1866, Hallé & 1863 -1919, Newton & 1878-1911, James \\
\hline 1856-1918, Eade & $1863-1899$, Stanley & 1878-1906, Teign- \\
\hline $1856-1884$, Knightley & 1864-1920, Apperley & mouth- \\
\hline $1856-1881$, Punshon & $1864-1894$, Barrow & Shore \\
\hline 1856 & $\begin{array}{r}\text { 1864-1920, Gladstone, } \\
\text { (Mrs.Drew) }\end{array}$ & $\begin{array}{l}\text { r879-1922, Cobden- } \\
\text { Sanderson }\end{array}$ \\
\hline
\end{tabular}




$\begin{array}{lll}\text { 1879-1891, Drummond } & \text { 1889-1914, Caddick } & \text { 1903-1919, Cummings } \\ \text { 1879-1900, Yerburgh } & \text { 1890-1902, Sturt } & \text { 1904-1915, Grenfell } \\ \text { 1880-1896, Holden } & \text { 1892-1903, McGregor } & \text { 1905-1948, Rich } \\ \text { 1880-1918, Meath } & \text { 1892-1946, Ruddy } & \text { 1906-1923, Hewins } \\ \text { 1881-1901, Gower } & \text { 1893-1923, Hewlett } & \text { 1906-1935, Williams } \\ \text { 1881-1932, Plunkett } & \text { 1893-1922, Wilson } & \text { 1908-1923, Riddell } \\ \text { 1882-1894, Bain } & \text { 1894-1934, Garraway } & \text { 1910-1932, Carr } \\ \text { 1883-1900, Collier } & \text { 1894-1935, Shilston } & \text { 1912-1922, Clarke } \\ \text { 1883-1938, Cornish } & \text { 1896-1928, Bennett } & \text { 1914-1929, Dobbs } \\ \text { 1883-1928, Haig } & \text { 1897-1925, Benson } & \text { 1916-1948, Burkinshaw } \\ \text { 1884-1924, Townshend } & \text { 1898-1909, Brown } & \text { 1916-1926, D'Abernon } \\ \text { 1884-1910, Walker } & \text { 1898-1922, Fitzroy } & \text { 1916-1944, Hall } \\ \text { 1886-1930, Bailey } & \text { 1898-1928, Ricketts } & \text { 1916-1930, Gregory } \\ \text { 1886-1948, , Cockerell } & \text { I899-1924, Rawlinson } & \text { 1916-1948, Sykes } \\ \text { 1887-1914, Blumenfeld } & \text { 1899-1917, Ypres } & \text { 1925-1944, Mattison } \\ \text { 1887-1902, Gissing } & \text { 1900-1913, Selous } & \text { 1930-1944, Collins } \\ \text { 1887-1942, Morton } & \text { 1901-1940, Brammer } & \text { 1932-1943, Agate } \\ \text { 1888-1914, Field } & \text { 1902-1933, Benson } & \text { 1937-1948, Crowley } \\ \text { 1888-1913, King-Hall } & & \end{array}$


